\title{
Pregnancy, breastfeeding and the SARS-CoV-2 vaccine: an ethics-based framework for shared decision-making
}

\author{
Jonathan S. Zipursky MD, Rebecca A Greenberg RN PhD, Cynthia Maxwell MD, Tali Bogler MD MScCH
}

— Cite as: CMAJ 2021 March 1;193:E312-4. doi: 10.1503/cmaj.202833; early-released January 27, 2021

D n December 2020, the Canadian National Advisory Committee on Immunization recommended that the vaccine against severe acute respiratory syndrome coronavirus 2 (SARS-CoV-2) should not be routinely offered to individuals who are pregnant or breastfeeding until further evidence is available, but can be considered in certain scenarios where the benefits are deemed to outweigh the risks. ${ }^{1}$ Guidance from specialist bodies in the United States, however, recommends against withholding the SARS-CoV-2 vaccination from individuals who are pregnant or breastfeeding. ${ }^{2,3}$ We propose that people who are pregnant (including those trying to conceive) or breastfeeding should be offered the SARS-CoV-2 vaccine on ethical grounds, and discuss how health care providers and patients can use a shared decision-making approach to guide these discussions.

Core principles of medical ethics hold that medical decisions or interventions should respect individuals' autonomy, be just, be beneficial (beneficence) and not cause harm (nonmaleficence). Excluding individuals who are pregnant or breastfeeding from accessing the SARS-CoV-2 vaccine limits autonomy and lacks consideration of individual factors. Pregnant individuals have the right to make decisions that are in their best interests, even when those interests may conflict with the well-being of their fetus. ${ }^{4}$ Categorical exclusion of this population does not allow individuals to make choices based on their values and personal circumstances. Despite the legal rights that pregnant individuals have in Canada, deep-rooted normative cultural assumptions that they should sacrifice their own health for the sake of their fetus may exist. Health equity considerations would value a parent's health as being equally important as that of their children or future children, and require that all people, pregnant or not, have equal access to the vaccine.

Existing data from clinical trials do not support the safety of the SARS-CoV-2 vaccine among individuals who are pregnant or breastfeeding; nor do they confirm that the vaccine harms the pregnant individual or fetus. We argue that withholding the vaccine is ethically justified only if clear, substantial and imminent maternal or fetal harms are expected. Although live virus

\section{KEY POINTS}

- Canadian guidance has recommended that the vaccine against severe acute respiratory syndrome coronavirus 2 (SARS-CoV-2) should not be routinely offered to individuals who are pregnant or breastfeeding until further evidence is available; however, it is ethically justified to offer individuals who are pregnant, trying to conceive or breastfeeding the option to receive the vaccine.

- Individuals who are pregnant or breastfeeding should be considered in distinct risk groups in discussions about the relative safety and benefit of the vaccine.

- Shared decision-making can help such individuals to make a choice that aligns with their own values, and health care workers should support the individual's decision to receive the vaccine or not.

- Factors to consider in patient-provider discussions include risk tolerance, personal risk of SARS-CoV-2 infection and associated morbidity and mortality; potential impact of the disease on the fetus and newborn; family and caregiver responsibilities; and the efficacy and safety of the vaccine for the pregnant individual and fetus.

vaccines are not generally recommended in pregnancy because of theoretical concerns regarding fetal infection, most other vaccines are considered safe. The mRNA vaccine technology used in the approved SARS-CoV-2 vaccines is novel, yet the findings of preliminary reproductive toxicology studies in animal models suggest they do not harm the fetus. ${ }^{5}$ Furthermore, among 23 individuals (12 in the vaccine arm, 11 in the placebo arm) who reported pregnancy after vaccination in the initial Pfizer-BioNTech vaccine trials, no adverse effects have been noted to date. ${ }^{6}$ In contrast, the findings of a living systematic review and analysis of surveillance data collected by the US Centers for Disease Control and Prevention suggest that symptomatic pregnant individuals with coronavirus disease 2019 (COVID-19) are at increased risk of severe illness and adverse pregnancy outcomes, including preterm birth. ${ }^{7,8}$ In addition, a recent statement from the Academy of Breastfeeding Medicine 
does not recommend cessation of breastfeeding in individuals who are vaccinated against SARS-CoV-2, as antibodies and $T$ cells stimulated by the vaccine might transfer into breast milk, thereby protecting the infant from SARS-CoV-2 infection. ${ }^{9}$ Preventing known risks of COVID-19, particularly in the face of limited evidence suggesting that health risks of the vaccine are few, would suggest that vaccination is likely beneficial to both the pregnant or breastfeeding individual and their fetus or child.

It is also important to consider an individual's likelihood of both acquiring SARS-CoV-2 infection and developing severe COVID-19 if they do. In Canada, as in many other countries, women are overrepresented in front-line health care and essential service jobs, placing them at disproportionately increased risk of SARS-CoV-2 infection. ${ }^{10}$ In addition, some individuals are at even higher risk of severe COVID-19 because of underlying obesity, diabetes or other comorbidities. ${ }^{7}$ If individuals are at high risk of illness, the argument that they should be protected is strengthened.

In Canada, individuals who are at high risk of contracting infection (e.g., health care workers) or are more vulnerable to the effects of the virus will be given priority access to the vaccine. ${ }^{11}$ Part of the justification for this is the concept of reciprocity: that individuals who knowingly put themselves in harm's way should have the option for protection. In addition, it will serve society to protect these individuals from SARS-CoV-2 infection - the concept of utility - as many health care workers perform essential front-line roles and cannot be easily replaced, because of their unique skill set. Restricting individuals who are pregnant or breastfeeding and who fall into this category shows a disregard for the risk they incur for others.

Until evidence emerges showing that harms outweigh benefits, we propose that all individuals who are pregnant or breastfeeding should be given the option of receiving the SARS-CoV-2 vaccine. Other options should also be presented as part of a shared decision-making approach, including delaying vaccination until further safety data are available, or forgoing vaccination altogether and continuing to follow public health measures to minimize the risk of SARS-CoV-2 infection.

Shared decision-making is a process whereby clinicians partner with patients to reach informed, value-laden medical decisions. ${ }^{12}$ Each individual who is pregnant or breastfeeding will fall into a distinct risk category and have their own personal considerations. Using a shared decision-making approach maximizes autonomy and allows every person to make a decision that fits with their values. Importantly, a patient should always feel supported in health decision-making. We therefore suggest use of a framework to support shared decision-making that allows individuals to weigh the risks and benefits, given the available evidence, and consider their personal values and circumstances. Patients will bring forward their values and goals to guide decision-making, and with input from their provider, can make a choice that is in their best interest. Some factors to consider in these discussions are outlined in Appendix 1 (available www.cmaj.ca/lookup/doi/10.1503/ cmaj.202833/tab-related-content), and include risk tolerance, personal risk of SARS-CoV-2 infection and associated morbidity and mortality, potential impact of COVID-19 on the fetus and newborn, family and caregiver responsibilities, and the efficacy and safety of the vaccine for the pregnant individual and fetus. Another important consideration may be the level of trust that the individual or community has in the health care system. Some groups, including Black and Indigenous people, may be hesitant about vaccination owing to long-standing racism and abuse. ${ }^{13}$

Discussions about the safety of the vaccine for individuals who are pregnant or breastfeeding will evolve over time as new safety data emerge. In some regions, these individuals can enrol in registries after receiving the vaccine and are expected to be included in additional vaccine trials in early 2021. In the interim, a permissive strategy is equitable, allowing individuals who are planning a pregnancy, are pregnant, or are breastfeeding to choose to receive the vaccine. This approach can be supported through shared decision-making with health care providers, which is in line with recent recommendations from the Society of Obstetricians and Gynaecologists of Canada. ${ }^{6}$

\section{References}

1. National Advisory Committee on Immunization. Recommendations on the use of COVID-19 vaccines. Ottawa: Government of Canada; 2021. Available: www. canada.ca/en/public-health/services/immunization/national-advisory-committee -on-immunization-naci/recommendations-use-covid-19-vaccines.html (accessed 2021 Jan. 11).

2. Riley LE, Beigi R, Jamieson DJ, et al. Vaccinating pregnant and lactating patients against COVID-19. Washington (D.C.): American College of Obstetricians and Gynecologists; 2020. Available: www.acog.org/clinical/clinical-guidance/practice -advisory/articles/2020/12/vaccinating-pregnant-and-lactating-patients-against -covid-19 (accessed 2020 Dec. 14)

3. Society for Maternal-Fetal Medicine (SMFM) statement: SARS-CoV-2 vaccination in pregnancy. Washington (D.C.): Society for Maternal Fetal Medicine; 2020:1-3. Available: www.smfm.org/publications/339-society-for-maternal-fetal -medicine-smfm-statement-sars-cov-2-vaccination-in-pregnancy (accessed 2020 Dec. 14).

4. Criminal Code of Canada, R.S.C., 1985, c. C-46, s.223 (1).

5. Vaccines and Related Biological Products Advisory Committee meeting December 17, 2020. FDA briefing document: Moderna COVID-19 vaccine. Silver Spring (MD): United States Food and Drug Administration; 2020.

6. SOGC statement on COVID-19 vaccination in pregnancy. Ottawa: The Society of Obstetricians and Gynaecologists of Canada; 2020. Available: www.sogc. org/en/content/featured-news/SOGC_Statement_on_COVID-19_Vaccination_ in_Pregnancy.aspx (accessed 2020 Dec. 17).

7. Allotey J, Stallings E, Bonet M, et al. Clinical manifestations, risk factors, and maternal and perinatal outcomes of coronavirus disease 2019 in pregnancy: living systematic review and meta-analysis. BMJ 2020;370:m3320. doi: 10.1136/bmj.m3320.

8. Zambrano LD, Ellington S, Strid P, et al. Update: characteristics of symptomatic women of reproductive age with laboratory-confirmed SARS-CoV-2 infection by pregnancy status - United States, January 22-October 3, 2020. MMWR Morb Mortal Wkly Rep 2020;69:1641-7.

9. Considerations for COVID-19 vaccination in lactation. Chicago: Academy of Breastfeeding Medicine; 2020. Available: www.bfmed.org/abm-statement -considerations-for-covid-19-vaccination-in-lactation (accessed 2020 Dec. 17).

10. Employment by class of worker, annual (x 1000): Table: 14-10-0027-01 (formerly CANSIM 282-0012). Ottawa: Statistics Canada. 2020. Available: www150.statcan .gc.ca/t1/tbl1/en/tv.action?pid=1410002701 (accessed 2020 Dec. 17).

11. Ismail SJ, Zhao L, Tunis MC, et al. Key populations for early COVID-19 immunization: preliminary guidance for policy. CMAJ 2020;192:E1620-32.

12. Grad R, Legare F, Bell NR, et al. Shared decision making in preventive health care What it is; what it is not. Can Fam Physician 2017;63:682-4.

13. Vohra-Miller S, Bhayana A. What good are COVID-19 vaccines if people are afraid? We need to build trust with racialized communities, specifically PSWs facing vaccine hesitancy. Toronto Star 2021 Jan. 8. 
Competing interests: None declared.

This article has been peer reviewed.

Affiliations: Department of Medicine (Zipursky), University of Toronto; Division of Clinical Pharmacology and Toxicology (Zipursky), Sunnybrook Health Sciences Centre; Bioethics Department (Greenberg), Sinai Health, Department of Paediatrics, University of Toronto; Department of Obstetrics and Gynaecology (Maxwell), Sinai Health; Department of Community and Family Medicine (Bogler), St. Michael's Hospital, Toronto, Ont.

Contributors: All of the authors contributed to the conception and design of the work, drafted the manuscript, revised it critically for important intellectual content, gave final approval of the version to be published and agreed to be accountable for all aspects of the work.
Content licence: This is an Open Access article distributed in accordance with the terms of the Creative Commons Attribution (CC BY-NC-ND 4.0) licence, which permits use, distribution and reproduction in any medium, provided that the original publication is properly cited, the use is noncommercial (i.e., research or educational use), and no modifications or adaptations are made. See: https://creativecommons.org/licenses/by-nc-nd/4.0/

Acknowledgements: The authors gratefully acknowledge Nipa Chauhan for creating the figure, and Nathan Stall for helpful comments on earlier versions of the manuscript.

Disclaimer: Tali Bogler is married to Nathan Stall, an associate editor of CMAJ, who was not involved with the editorial decision-making process for this article.

Correspondence to: Jonathan Zipursky, Jonathan.Zipursky@sunnybrook.ca 